\title{
On Modules over Local Rings
}

\author{
Fatma Özen Erdoğan, Süleyman Çiftçi and Atilla Akpınar
}

\begin{abstract}
This paper is dealed with a special local ring $\mathbf{A}$ and modules over $\mathbf{A}$. Some properties of modules, that are constructed over the real plural algebra, are investigated. Moreover a module is constructed over the linear algebra of matrix $M_{m m}(\mathbb{R})$ and one of its basis is found.
\end{abstract}

\section{Introduction.}

The structure of a field which has many simplicities in its operations, in fact is a generalization of the system of real numbers $\mathbb{R}$. The structure of a ring which does not have some properties that a field has, is also a generalization of the system of integers $\mathbb{Z}$. For example, while all linear equations have solutions according to both addition and multiplication in $\mathbb{R}$ or in a field, every linear equation according to multiplication in $\mathbb{Z}$ or in a ring does not necessarily have any solutions because of non-existence of inverse element. In the study of vector spaces constructed over fields, certainly there are many simplicities compared to the study of modules constructed over rings. Modules are more general structures than vector spaces. For more detailed study on modules, we refer to [1]. For the Algebra and Linear Algebra that will be used throughout this paper, we refer to [4] and [5].

In this paper, we investigate some properties of modules constructed over the real plural algebra $\boldsymbol{A}$. We construct a module over the linear algebra of matrix $M_{m m}(\mathbb{R})$ and we find one of its basis. Furthermore, we give a theorem that describes the linear independent vectors in a module constructed over a local ring.

Key Words: reel plural algebra, local ring, module.

2010 Mathematics Subject Classification: 13C99.

Received: 15.01.2014.

Accepted: 02.07.2014. 


\section{Preliminaries.}

In this section, we will recall some basic definitons and propositions from [2] and [3].

Definition 1 ([2, Def. 1.1]). A real plural algebra of order $m$ is a linear algebra $\boldsymbol{A}$ on $\mathbb{R}$ having, as a vector space over $\mathbb{R}$, a basis $\left\{1, \eta, \eta^{2}, \eta^{3}, \cdots, \eta^{m-1}\right\}$, where $\eta^{m}=0$.

A ring with identity element is called local if the set of its non-units form an ideal. A module that is constructed over a local ring $\boldsymbol{A}$ is called an $\boldsymbol{A}$-module.

Definition 2 ([3, Def. 1]). Let $\boldsymbol{A}$ be a local ring. Let $M$ be a finitely generated $\boldsymbol{A}$-module. Then $\boldsymbol{M}$ is an $\boldsymbol{A}$-space of finite dimension if there exists $E_{1}, E_{2}, \cdots, E_{n}$ in $M$ with

i) $M=\boldsymbol{A} E_{1} \oplus \boldsymbol{A} E_{2} \oplus \cdots \oplus \boldsymbol{A} E_{n}$

ii) the map $\boldsymbol{A} \rightarrow \boldsymbol{A} E_{i}$ defined by $x \rightarrow x E_{i}$ is an isomorphism for $1 \leq i \leq n$.

Definition 3 ([2, Def. 1.2]). By a system of projections $\boldsymbol{A} \rightarrow \mathbb{R}$, it is meant a system of mappings $p_{k}: \boldsymbol{A}$ onto $\mathbb{R}$, defined for $k=0,1, \cdots, m-1$, as follows:

$$
\forall \beta \in \boldsymbol{A}, \beta=\sum_{i=0}^{m-1} b_{i} \eta^{i}, p_{k}(\beta):=b_{k} .
$$

Now we can introduce the following propositions without proof, from [2].

Proposition 4 ([2, Prop. 1.3]). An element $\varepsilon \in \boldsymbol{A}$ is a unit if and only if $p_{0}(\varepsilon) \neq 0$.

Proposition 5 ([2, Prop. 1.5]). $\boldsymbol{A}$ is a local ring with the maximal ideal $\eta \boldsymbol{A}$. The ideals $\eta^{j} \boldsymbol{A}, 1 \leq j \leq m$, are all ideals in $\boldsymbol{A}$.

\section{Modules Constructed Over The Real Plural Algebra.}

In this section, we examine some properties of modules constructed over the real plural algebra $\boldsymbol{A}$. First we give a theorem that tells us the relation between units and zero divisors in $\boldsymbol{A}$. Then we give a short proof of the isomorphism between $\boldsymbol{A}$ and linear algebra of matrix $\boldsymbol{K}=M_{m m}(\mathbb{R})$. After that we investigate a basis for $\boldsymbol{K}$ and we construct a module over $\boldsymbol{K}$ and find one of its basis. We give a theorem that describes the linear independent vectors in a module constructed over a local ring. Finally we give a detailed proof of Remark 2 given in [3]. 
Theorem 6. None of the units of $\boldsymbol{A}$ are zero divisors, namely for every $\alpha, \beta \in \boldsymbol{A}$;

$$
\alpha=\sum_{i=0}^{m-1} a_{i} \eta^{i}, a_{0} \neq 0 \quad \text { and } \beta=\sum_{i=0}^{m-1} b_{i} \eta^{i} \text { if } \alpha \cdot \beta=0 \text { or } \beta \cdot \alpha=0 \text {, }
$$

then $\beta=0$. Also for $1 \leq k \leq m-1$ and $\alpha=a_{k} \eta^{k}+a_{k+1} \eta^{k+1}+\cdots+a_{m-1} \eta^{m-1}$ if $\alpha \cdot \beta=0$ or $\beta \cdot \alpha=0$, then $\beta=b_{m-k} \eta^{m-k}+b_{m-k+1} \eta^{m-k+1}+\cdots+b_{m-1} \eta^{m-1}$.

Proof. If $\alpha$ is a unit, then there is an inverse element $\alpha^{-1}$ and since there is an associative property in the real algebra;

$$
\alpha \cdot \beta=0 \Rightarrow \alpha^{-1}(\alpha \cdot \beta)=\alpha^{-1} \cdot 0 \Rightarrow \beta=0 .
$$

For $\beta \cdot \alpha=0$, it is easily seen that $\beta=0$ by similar calculations.

Now we show that for $1 \leq k \leq m-1, \alpha \cdot \beta=\left(a_{k} \eta^{k}+a_{k+1} \eta^{k+1}+\cdots+\right.$ $\left.a_{m-1} \eta^{m-1}\right)\left(b_{0}+b_{1} \eta+b_{2} \eta^{2}+\cdots+b_{m-1} \eta^{m-1}\right)=0 \Rightarrow \beta=b_{m-k} \eta^{m-k}+$ $b_{m-k+1} \eta^{m-k+1}+\cdots+b_{m-1} \eta^{m-1}$.

First let $k=1$, so we deal with $\alpha=a_{1} \eta+a_{2} \eta^{2}+\cdots+a_{m-1} \eta^{m-1}$. We have $\alpha \cdot \beta=\left(a_{1} \eta+a_{2} \eta^{2}+\cdots+a_{m-1} \eta^{m-1}\right) \cdot\left(b_{0}+b_{1} \eta+b_{2} \eta^{2}+\cdots+b_{m-1} \eta^{m-1}\right)$ and

$$
\begin{aligned}
& \alpha \cdot \beta=0 \\
& \Rightarrow\left(a_{1} b_{0}\right) \eta+\left(a_{1} b_{1}+a_{2} b_{0}\right) \eta^{2}+\left(a_{1} b_{2}+a_{2} b_{1}+a_{3} b_{0}\right) \eta^{3} \\
& +\cdots+\left(a_{1} b_{m-2}+a_{2} b_{m-3}+\cdots+a_{m-2} b_{1}+a_{m-1} b_{0}\right) \eta^{m-1} \\
& +\left(a_{1} b_{m-1}+a_{2} b_{m-2}+\cdots+a_{m-2} b_{2}+a_{m-1} b_{1}\right) \eta^{m} \\
& +\left(a_{2} b_{m-1}+a_{3} b_{m-2}+\cdots+a_{m-2} b_{3}+a_{m-1} b_{2}\right) \eta^{m+1} \\
& +\cdots+\left(a_{m-1} b_{m-1}\right) \eta^{2 m-2} \\
& =0 \eta+0 \eta^{2}+\cdots+0 \eta^{m-1}+c_{m} \eta^{m}+c_{m+1} \eta^{m+1}+\cdots+c_{2 m-2} \eta^{2 m-2} .
\end{aligned}
$$

Here since $\eta^{m}=\eta^{m+1}=\cdots=\eta^{2 m-2}=0$, the coefficients $c_{m}, c_{m+1}, \cdots, c_{2 m-2}$ need not to be zero. Forcing the coefficient of $\eta$ to be zero, we obtain $a_{1} b_{0}=0$, and since $a_{1} \neq 0$, we find $b_{0}=0$. Forcing the coefficient of $\eta^{2}$ to be zero, we obtain $a_{1} b_{1}+a_{2} b_{0}=0$. If we put $b_{0}=0$ in this equation and use the fact that $a_{1} \neq 0$, we obtain $b_{1}=0$. Forcing the coefficient of $\eta^{3}$ to be zero, we get $a_{1} b_{2}+a_{2} b_{1}+a_{3} b_{0}=0$. Putting $b_{0}=b_{1}=0$ in this equation and using $a_{1} \neq 0$, we find $b_{2}=0$. Carrying on this process, we get $b_{k}=0$ for $0 \leq k \leq m-3$. Finally forcing the coefficient of $\eta^{m-1}$ to be zero, we get $a_{1} b_{m-2}+a_{2} b_{m-3}+\cdots+a_{m-2} b_{1}+a_{m-1} b_{0}=0$. Putting $b_{0}=b_{1}=\cdots=b_{m-3}=0$ in this equation and using $a_{1} \neq 0$, we find $b_{m-2}=0$. If we put $b_{0}=b_{1}=\cdots=b_{m-3}=b_{m-2}=0$ in the following equation, we have $\left(a_{1} b_{m-1}\right) \eta^{m}=0$. Since $\eta^{m}=0$, then $b_{m-1} \in \mathbb{R}$. Thus for $k=1$, we obtain 
$\alpha \cdot \beta=\left(a_{1} \eta+a_{2} \eta^{2}+\cdots+a_{m-1} \eta^{m-1}\right) \cdot\left(b_{0}+b_{1} \eta+b_{2} \eta^{2}+\cdots+b_{m-1} \eta^{m-1}\right)=$ $0 \Rightarrow \beta=b_{m-1} \eta^{m-1}$.

Now let $k=2$ so we deal with $\alpha=a_{2} \eta^{2}+a_{3} \eta^{3}+\cdots+a_{m-1} \eta^{m-1}$. We have $\alpha \cdot \beta=\left(a_{2} \eta^{2}+a_{3} \eta^{3}+\cdots+a_{m-1} \eta^{m-1}\right) \cdot\left(b_{0}+b_{1} \eta+b_{2} \eta^{2}+\cdots+b_{m-1} \eta^{m-1}\right)$ and

$$
\begin{aligned}
& \alpha \cdot \beta=0 \\
& \Rightarrow\left(a_{2} b_{0}\right) \eta^{2}+\left(a_{2} b_{1}+a_{3} b_{0}\right) \eta^{3}+\left(a_{2} b_{2}+a_{3} b_{1}+a_{4} b_{0}\right) \eta^{4} \\
& +\cdots+\left(a_{2} b_{m-4}+a_{3} b_{m-5}+\cdots+a_{m-3} b_{1}+a_{m-2} b_{0}\right) \eta^{m-2} \\
& +\left(a_{2} b_{m-3}+a_{3} b_{m-4}+\cdots+a_{m-2} b_{1}+a_{m-1} b_{0}\right) \eta^{m-1} \\
& +\left(a_{2} b_{m-2}+a_{3} b_{m-3}+\cdots+a_{m-2} b_{2}+a_{m-1} b_{1}\right) \eta^{m} \\
& +\left(a_{2} b_{m-1}+a_{3} b_{m-2}+\cdots+a_{m-2} b_{3}+a_{m-1} b_{2}\right) \eta^{m+1} \\
& +\cdots+\left(a_{m-1} b_{m-1}\right) \eta^{2 m-2} \\
& =0 \eta+0 \eta^{2}+\cdots+0 \eta^{m-1}+c_{m} \eta^{m}+c_{m+1} \eta^{m+1}+\cdots+c_{2 m-2} \eta^{2 m-2} .
\end{aligned}
$$

Here since $\eta^{m}=\eta^{m+1}=\cdots=\eta^{2 m-2}=0$, the coefficients $c_{m}, c_{m+1}, \cdots, c_{2 m-2}$ need not be zero. Forcing the coefficient of $\eta^{2}$ to be zero, we obtain $a_{2} b_{0}=0$ and since $a_{2} \neq 0$, we find $b_{0}=0$. Forcing the coefficient of $\eta^{3}$ to be zero, we obtain $a_{2} b_{1}+a_{3} b_{0}=0$. If we use $b_{0}=0$ in this equation and use the fact that $a_{2} \neq 0$, we obtain $b_{1}=0$. Forcing the coefficient of $\eta^{4}$ to be zero, we get $a_{2} b_{2}+a_{3} b_{1}+a_{4} b_{0}=0$. If we put $b_{0}=b_{1}=0$ in this equation and use $a_{2} \neq 0$, we obtain $b_{2}=0$. Continuing on this process, we get $b_{k}=0$ for $0 \leq k \leq m-3$. If we put $b_{0}=b_{1}=\cdots=b_{m-3}=0$ in the coefficient of $\eta^{m}$, we have $\left(a_{2} b_{m-2}\right) \eta^{m}=0$. Since $\eta^{m}=0$, we find $b_{m-2} \in \mathbb{R}$. Similarly if we put $b_{0}=b_{1}=\cdots=b_{m-3}=0$ and $b_{m-2} \in \mathbb{R}$, in the coefficient of $\eta^{m+1}$, we get $\left(a_{2} b_{m-1}+a_{3} b_{m-2}\right) \eta^{m+1}=0$. Since $\eta^{m+1}=0$, we have $b_{m-1} \in \mathbb{R}$. Thus for $k=2$, we find $\alpha \cdot \beta=\left(a_{2} \eta^{2}+a_{3} \eta^{3}+\cdots+a_{m-1} \eta^{m-1}\right) \cdot\left(b_{0}+b_{1} \eta+b_{2} \eta^{2}+\right.$ $\left.\cdots+b_{m-1} \eta^{m-1}\right)=0 \Rightarrow \beta=b_{m-2} \eta^{m-2}+b_{m-1} \eta^{m-1}$.

Finally we generalize this process for $k$. Let $\alpha=a_{k} \eta^{k}+a_{k+1} \eta^{k+1}+\cdots+$ $a_{m-1} \eta^{m-1}$. Then we have

$$
\alpha \cdot \beta=\left(a_{k} \eta^{k}+a_{k+1} \eta^{k+1}+\cdots+a_{m-1} \eta^{m-1}\right) \cdot\left(b_{0}+b_{1} \eta+b_{2} \eta^{2}+\cdots+b_{m-1} \eta^{m-1}\right)
$$
and

$$
\begin{aligned}
& \alpha \cdot \beta=0 \\
& \Rightarrow\left(a_{k} b_{0}\right) \eta^{k}+\left(a_{k} b_{1}+a_{k+1} b_{0}\right) \eta^{k+1}+\left(a_{k} b_{2}+a_{k+1} b_{1}+a_{k+2} b_{0}\right) \eta^{k+2}
\end{aligned}
$$




$$
\begin{aligned}
& +\cdots+\left(a_{k} b_{m-(k+1)}+a_{k+1} b_{m-(k+2)}+\cdots+a_{m-1} b_{0}\right) \eta^{m-1} \\
& +\left(a_{k} b_{m-k}+a_{k+1} b_{m-(k+1)}+\cdots+a_{m-2} b_{2}+a_{m-1} b_{1}\right) \eta^{m} \\
& +\left(a_{k} b_{m-(k-1)}+a_{k+1} b_{m-k}+\cdots+a_{m-2} b_{3}+a_{m-1} b_{2}\right) \eta^{m+1} \\
& +\cdots+\left(a_{m-1} b_{m-1}\right) \eta^{2 m-2} \\
& =0 \eta+0 \eta^{2}+\cdots+0 \eta^{m-1}+c_{m} \eta^{m}+c_{m+1} \eta^{m+1}+\cdots+c_{2 m-2} \eta^{2 m-2} .
\end{aligned}
$$

Here since $\eta^{m}=\eta^{m+1}=\cdots=\eta^{2 m-2}=0$, the coefficients $c_{m}, c_{m+1}, \cdots, c_{2 m-2}$ need not be zero. Forcing the coefficient of $\eta^{k}$ to be zero, we obtain $a_{k} b_{0}=0$ and since $a_{k} \neq 0$, we find $b_{0}=0$. Forcing the coefficient of $\eta^{k+1}$ to be zero, we obtain $a_{k} b_{1}+a_{k+1} b_{0}=0$. If we put $b_{0}=0$ in this equation and use $a_{k} \neq 0$, we obtain $b_{1}=0$. Forcing the coefficient of $\eta^{k+2}$ to be zero, we get $a_{k} b_{2}+$ $a_{k+1} b_{1}+a_{k+2} b_{0}=0$. If we use $b_{0}=b_{1}=0$ in this last equation and use $a_{k} \neq 0$, we obtain $b_{2}=0$. Carrying on this process, we can easily find $b_{0}=b_{1}=\cdots=$ $b_{m-(k+1)}=0$ by forcing the coefficients of $\eta^{k}, \eta^{k+1}, \eta^{k+2}, \cdots, \eta^{m-1}$ to be zero. Now if we put $b_{0}=b_{1}=\cdots=b_{m-(k+1)}=0$ in the coefficient of $\eta^{m}$, we obtain $\left(a_{k} b_{m-k}\right) \eta^{m}=0$. Since $\eta^{m}=0$, we have $b_{m-k} \in \mathbb{R}$. By similar calculations, if we use $b_{0}=b_{1}=\cdots=b_{m-(k+1)}=0$ and $b_{m-k} \in \mathbb{R}$ in the coefficient of $\eta^{m+1}$, we obtain $\left(a_{k} b_{m-(k-1)}+a_{k+1} b_{m-k}\right) \eta^{m+1}=0$. Since $\eta^{m+1}=0$, we get $b_{m-(k-1)} \in \mathbb{R}$. Carrying on this process by using $\eta^{m}=\eta^{m+1}=\cdots=\eta^{2 m-2}=$ 0 we can easily find $b_{m-k}, b_{m-(k-1)}, b_{m-(k-2)}, \cdots, b_{m-1} \in \mathbb{R}$. Thus for $\alpha=$ $a_{k} \eta^{k}+a_{k+1} \eta^{k+1}+\cdots+a_{m-1} \eta^{m-1}$ and $\beta=b_{0}+b_{1} \eta+b_{2} \eta^{2}+\cdots+b_{m-1} \eta^{m-1}$, $\alpha \cdot \beta=0$ implies $\beta=b_{m-k} \eta^{m-k}+b_{m-k+1} \eta^{m-k+1}+\cdots+b_{m-1} \eta^{m-1}$.

Let $\boldsymbol{A}$ be a real plural algebra having a basis $\left\{1, \eta, \eta^{2}, \eta^{3}, \cdots, \eta^{m-1}\right\}$ with $\eta^{m}=0$. Let $\boldsymbol{K}=M_{m m}(\mathbb{R})$ be the linear algebra of matrix of the form

$$
\left(\begin{array}{cccc}
b_{0} & b_{1} & \cdots & b_{m-1} \\
0 & b_{0} & \cdots & b_{m-2} \\
\vdots & \vdots & \ddots & \vdots \\
0 & \cdots & \cdots & b_{0}
\end{array}\right),
$$

where $b_{i} \in \mathbb{R}$ for $0 \leq i \leq m-1$. In [2] it is stated that without proof the map $f: \boldsymbol{A} \rightarrow \boldsymbol{K}$ which is given as

$$
f(\alpha)=\left(a_{i j}\right)= \begin{cases}a_{i j}=0, & j<i \\ a_{i j}=a_{j-i}, & j \geq i .\end{cases}
$$

for every $\alpha=\sum_{i=0}^{m-1} a_{i} \eta^{i} \in \boldsymbol{A}$ is isomorphism. Here we give the short proof of this proposition. 
Proposition 7. The ring $\boldsymbol{A}$ is isomorphic to the linear algebra of matrix $\boldsymbol{K}$ of the form:

$$
\left(\begin{array}{cccc}
b_{0} & b_{1} & \cdots & b_{m-1} \\
0 & b_{0} & \cdots & b_{m-2} \\
\vdots & \vdots & \ddots & \vdots \\
0 & \cdots & \cdots & b_{0}
\end{array}\right) .
$$

Proof. The map $f: \boldsymbol{A} \rightarrow \boldsymbol{K}$ is defined in the following way: For every $\alpha=$ $\sum_{i=0}^{m-1} a_{i} \eta^{i} \in \boldsymbol{A}$

Then $f(\alpha)=\left(\begin{array}{ccccc}a_{0} & a_{1} & a_{2} & \cdots & a_{m-1} \\ 0 & a_{0} & a_{1} & \cdots & a_{m-2} \\ 0 & 0 & a_{0} & \cdots & a_{m-3} \\ \vdots & \vdots & \vdots & \ddots & \vdots \\ 0 & 0 & 0 & \cdots & a_{0}\end{array}\right)$.

Now for the considered mapping $f$, it is easily seen that $f$ is one to one, onto and for every $\alpha=a_{0}+a_{1} \eta+a_{2} \eta^{2}+\cdots+a_{m-1} \eta^{m-1}$ and $\beta=b_{0}+b_{1} \eta+$ $b_{2} \eta^{2}+\ldots+b_{m-1} \eta^{m-1} \in \boldsymbol{A}$ and for every $c \in \mathbb{R}$

$$
f(\alpha+\beta)=f(\alpha)+f(\beta) \text { and } f(c \alpha)=c f(\alpha) .
$$

Now we show the multiplication are preserved under this map. For every $\alpha$, $\beta \in \boldsymbol{A}, \alpha \cdot \beta=\left(a_{0} b_{0}\right)+\left(a_{0} b_{1}+a_{1} b_{0}\right) \eta+\left(a_{0} b_{2}+a_{1} b_{1}+a_{2} b_{0}\right) \eta^{2}+. \cdots+\left(a_{0} b_{m-1}+\right.$ $\left.a_{1} b_{m-2}+\cdots+a_{m-1} b_{0}\right) \eta^{m-1}$. Then we obtain

$$
f(\alpha \beta)=\left(\begin{array}{cccc}
a_{0} b_{0} & a_{0} b_{1}+a_{1} b_{0} & \cdots & a_{0} b_{m-1}+a_{1} b_{m-2}+\cdots+a_{m-1} b_{0} \\
0 & a_{0} b_{0} & \cdots & a_{0} b_{m-2}+a_{1} b_{m-3}+\cdots+a_{m-2} b_{0} \\
0 & 0 & \ddots & a_{0} b_{m-3}+a_{1} b_{m-2}+\cdots+a_{m-3} b_{0} \\
\vdots & \vdots & \ddots & \vdots \\
0 & 0 & \cdots & a_{0} b_{0}
\end{array}\right)
$$

and by using multiplication of matrices, we get

$$
\begin{aligned}
f(\alpha \cdot \beta) & =\left(\begin{array}{ccccc}
a_{0} & a_{1} & a_{2} & \cdots & a_{m-1} \\
0 & a_{0} & a_{1} & \cdots & a_{m-2} \\
0 & 0 & a_{0} & \cdots & a_{m-3} \\
\vdots & \vdots & \vdots & \ddots & \vdots \\
0 & 0 & 0 & \cdots & a_{0}
\end{array}\right) \cdot\left(\begin{array}{ccccc}
b_{0} & b_{1} & b_{2} & \cdots & b_{m-1} \\
0 & b_{0} & b_{1} & \cdots & b_{m-2} \\
0 & 0 & b_{0} & \cdots & b_{m-3} \\
\vdots & \vdots & \vdots & \ddots & \vdots \\
0 & 0 & 0 & \cdots & b_{0}
\end{array}\right) \\
& =f(\alpha) \cdot f(\beta) .
\end{aligned}
$$


Now we investigate one of the basis of $\boldsymbol{K}$. If we take any element of $\boldsymbol{K}$ such that

$$
B=\left(\begin{array}{ccccc}
b_{0} & b_{1} & b_{2} & \cdots & b_{m-1} \\
0 & b_{0} & b_{1} & \cdots & b_{m-2} \\
0 & 0 & b_{0} & \cdots & b_{m-3} \\
\vdots & \vdots & \vdots & \ddots & \vdots \\
0 & 0 & 0 & \cdots & b_{0}
\end{array}\right) \in \boldsymbol{K}
$$

we can write it as follows:

$$
\begin{gathered}
B=\left(\begin{array}{ccccc}
b_{0} & b_{1} & b_{2} & \cdots & b_{m-1} \\
0 & b_{0} & b_{1} & \cdots & b_{m-2} \\
0 & 0 & b_{0} & \cdots & b_{m-3} \\
\vdots & \vdots & \vdots & \ddots & \vdots \\
0 & 0 & 0 & \cdots & b_{0}
\end{array}\right)=b_{0}\left(\begin{array}{ccccc}
1 & 0 & 0 & \cdots & 0 \\
0 & 1 & 0 & \cdots & 0 \\
0 & 0 & 1 & \cdots & 0 \\
\vdots & \vdots & \vdots & \ddots & \vdots \\
0 & 0 & 0 & \cdots & 1
\end{array}\right) \\
+b_{1}\left(\begin{array}{ccccc}
0 & 1 & 0 & \cdots & 0 \\
0 & 0 & 1 & \cdots & 0 \\
0 & 0 & 0 & \ddots & 0 \\
\vdots & \vdots & \vdots & \ddots & 1 \\
0 & 0 & 0 & \cdots & 0
\end{array}\right)+\cdots+b_{m-1}\left(\begin{array}{ccccc}
0 & 0 & 0 & \cdots & 1 \\
0 & 0 & 0 & \cdots & 0 \\
0 & 0 & 0 & \ddots & 0 \\
\vdots & \vdots & \vdots & \ddots & 0 \\
0 & 0 & 0 & \cdots & 0
\end{array}\right) .
\end{gathered}
$$

Here if we put

$$
\begin{aligned}
& \eta_{0}=\left(a_{i j}\right)=\left(\begin{array}{ccccc}
1 & 0 & 0 & \cdots & 0 \\
0 & 1 & 0 & \cdots & 0 \\
0 & 0 & 1 & \cdots & 0 \\
\vdots & \vdots & \vdots & \ddots & \vdots \\
0 & 0 & 0 & \cdots & 1
\end{array}\right)_{m \times m} \quad \text { where } a_{i j}= \begin{cases}1, & i=j \\
0, & i \neq j\end{cases} \\
& \eta_{1}=\left(a_{i j}\right)=\left(\begin{array}{ccccc}
0 & 1 & 0 & \cdots & 0 \\
0 & 0 & 1 & \cdots & 0 \\
0 & 0 & 0 & \ddots & 0 \\
\vdots & \vdots & \vdots & \ddots & 1 \\
0 & 0 & 0 & \cdots & 0
\end{array}\right)_{m \times m} \text { where } a_{i j}= \begin{cases}1, & j=i+1 \\
0, & j \neq i+1\end{cases} \\
& \eta_{2}=\left(a_{i j}\right)= \\
& \left.\begin{array}{ccccc}
0 & 0 & 1 & \cdots & 0 \\
0 & 0 & 0 & \ddots & 0 \\
0 & 0 & 0 & \ddots & 1 \\
\vdots & \vdots & \vdots & \ddots & 0 \\
0 & 0 & 0 & \cdots & 0
\end{array}\right)_{m \times m} \text { where } a_{i j}= \begin{cases}1, & j=i+2 \\
0, & j \neq i+2\end{cases}
\end{aligned}
$$




$$
\eta_{m-1}=\left(a_{i j}\right)=\left(\begin{array}{ccccc}
0 & 0 & 0 & \cdots & 1 \\
0 & 0 & 0 & \cdots & 0 \\
0 & 0 & 0 & \ddots & 0 \\
\vdots & \vdots & \vdots & \ddots & 0 \\
0 & 0 & 0 & \cdots & 0
\end{array}\right) \text { where } a_{i j}=\left\{\begin{array}{cc}
1, & j=i+(m-1) \\
0, & j \neq i+(m-1)
\end{array}\right.
$$

then it is seen that $B=b_{0} \eta_{0}+b_{1} \eta_{1}+b_{2} \eta_{2}+\cdots+b_{m-1} \eta_{m-1}$ and also linear independence of the set $\left\{\eta_{0}, \eta_{1}, \eta_{2}, \cdots, \eta_{m-1}\right\}$ is obvious. Thus the set $\left\{\eta_{0}, \eta_{1}, \eta_{2}, \cdots, \eta_{m-1}\right\}$ is a basis of $\boldsymbol{K}$. We can express any element of this set in general as follows:

For $0 \leq i, j, k \leq m-1, \eta_{k}=\left(a_{i j}\right)_{m \times m}$ where $a_{i j}=\left\{\begin{array}{ll}1, & j=i+k \\ 0, & j \neq i+k\end{array}\right.$.

Also if we take

$$
\eta=\eta_{1}=\left(\begin{array}{ccccc}
0 & 1 & 0 & \cdots & 0 \\
0 & 0 & 1 & \cdots & 0 \\
0 & 0 & 0 & \ddots & 0 \\
\vdots & \vdots & \vdots & \ddots & 1 \\
0 & 0 & 0 & \cdots & 0
\end{array}\right)
$$

we see that $\eta_{k}=\eta^{k}$ for every $\eta_{k}$ where $1 \leq k \leq m-1$. For example

$$
\begin{aligned}
\eta^{2}= & \left(\begin{array}{ccccc}
0 & 1 & 0 & \cdots & 0 \\
0 & 0 & 1 & \cdots & 0 \\
0 & 0 & 0 & \ddots & 0 \\
\vdots & \vdots & \vdots & \ddots & 1 \\
0 & 0 & 0 & \cdots & 0
\end{array}\right)\left(\begin{array}{ccccc}
0 & 1 & 0 & \cdots & 0 \\
0 & 0 & 1 & \cdots & 0 \\
0 & 0 & 0 & \ddots & 0 \\
\vdots & \vdots & \vdots & \ddots & 1 \\
0 & 0 & 0 & \cdots & 0
\end{array}\right) \\
= & \left(\begin{array}{ccccc}
0 & 0 & 1 & \cdots & 0 \\
0 & 0 & 0 & \ddots & 0 \\
0 & 0 & 0 & \ddots & 1 \\
\vdots & \vdots & \vdots & \ddots & 0 \\
0 & 0 & 0 & \cdots & 0
\end{array}\right)=\eta_{2} .
\end{aligned}
$$

Now we construct a module $M$ over the linear algebra of matrix $\boldsymbol{K}$, then we will find one of the basis of it.

Proposition 8. $M=\mathbb{R}_{n}^{m}$ is a module over the linear algebra of matrix $\boldsymbol{K}$. Then the following set is a basis of $\boldsymbol{K}$-module $M$. 


$$
\begin{aligned}
& \left\{E_{1}=\left(\begin{array}{ccccc}
0 & 0 & 0 & \cdots & 0 \\
0 & 0 & 0 & \cdots & 0 \\
\vdots & \vdots & \vdots & \ddots & \vdots \\
1 & 0 & 0 & \cdots & 0
\end{array}\right), E_{2}=\left(\begin{array}{ccccc}
0 & 0 & 0 & \cdots & 0 \\
0 & 0 & 0 & \cdots & 0 \\
\vdots & \vdots & \vdots & \ddots & \vdots \\
0 & 1 & 0 & \cdots & 0
\end{array}\right)\right. \\
& \left.E_{3}=\left(\begin{array}{ccccc}
0 & 0 & 0 & \cdots & 0 \\
0 & 0 & 0 & \cdots & 0 \\
\vdots & \vdots & \vdots & \ddots & \vdots \\
0 & 0 & 1 & 0 \cdots & 0
\end{array}\right), \cdots, E_{n}=\left(\begin{array}{ccccc}
0 & 0 & 0 & \cdots & 0 \\
0 & 0 & 0 & \cdots & 0 \\
\vdots & \vdots & \vdots & \ddots & \vdots \\
0 & 0 & 0 & \cdots & 1
\end{array}\right)\right\}
\end{aligned}
$$

Proof. Linear independence of this set is obvious. Moreover for every $X \in M$, $X$ can be written as follows:

$$
\begin{aligned}
& X=\left(\begin{array}{ccccc}
x_{11} & x_{12} & x_{13} & \cdots & x_{1 n} \\
x_{21} & x_{22} & x_{23} & \cdots & x_{2 n} \\
\vdots & \vdots & \vdots & \ddots & \vdots \\
x_{m 1} & x_{m 2} & x_{m 3} & \cdots & x_{m n}
\end{array}\right) \\
& =\left(\begin{array}{cccc}
x_{m 1} & x_{(m-1) 1} & \cdots & x_{11} \\
0 & x_{m 1} & \cdots & x_{21} \\
\vdots & 0 & \ddots & \vdots \\
0 & \cdots & 0 & x_{m 1}
\end{array}\right)\left(\begin{array}{ccccc}
0 & 0 & 0 & \cdots & 0 \\
0 & 0 & 0 & \cdots & 0 \\
\vdots & \vdots & \vdots & \ddots & \vdots \\
1 & 0 & 0 & \cdots & 0
\end{array}\right) \\
& +\left(\begin{array}{cccc}
x_{m 2} & x_{(m-1) 2} & \cdots & x_{12} \\
0 & x_{m 2} & \cdots & x_{22} \\
\vdots & 0 & \ddots & \vdots \\
0 & \cdots & 0 & x_{m 2}
\end{array}\right)\left(\begin{array}{ccccc}
0 & 0 & 0 & \cdots & 0 \\
0 & 0 & 0 & \cdots & 0 \\
\vdots & \vdots & \vdots & \ddots & \vdots \\
0 & 1 & 0 & \cdots & 0
\end{array}\right) \\
& +\cdots+\left(\begin{array}{cccc}
x_{m n} & x_{(m-1) n} & \cdots & x_{1 n} \\
0 & x_{m n} & \cdots & x_{2 n} \\
\vdots & 0 & \ddots & \vdots \\
0 & \cdots & 0 & x_{m n}
\end{array}\right)\left(\begin{array}{ccccc}
0 & 0 & 0 & \cdots & 0 \\
0 & 0 & 0 & \cdots & 0 \\
\vdots & \vdots & \vdots & \ddots & \vdots \\
0 & 0 & 0 & \cdots & 1
\end{array}\right)
\end{aligned}
$$

Thus $\left[E_{1}, E_{2}, \cdots, E_{n}\right]=M$. Consequently, the set $\left\{E_{1}, E_{2}, \cdots, E_{n}\right\}$ is a basis of $\boldsymbol{K}$-module $M$.

For some $a_{1}, a_{2}, \cdots, a_{n} \in \mathbb{R}$, we write

$$
a_{1} E_{1}+a_{2} E_{2}+\cdots+a_{n} E_{n}=\left(\begin{array}{ccccc}
0 & 0 & 0 & \cdots & 0 \\
0 & 0 & 0 & \cdots & 0 \\
\vdots & \vdots & \vdots & \ddots & 0 \\
a_{1} & a_{2} & a_{3} & \cdots & a_{n}
\end{array}\right) .
$$


So if $M$ is thought as a vector space over $\mathbb{R}$, it is seen that

$$
\begin{aligned}
{\left[E_{1}, E_{2}, \cdots, E_{n}\right] } & =P_{0} \\
& =\left\{\left(\begin{array}{ccccc}
0 & 0 & 0 & \cdots & 0 \\
0 & 0 & 0 & \cdots & 0 \\
\vdots & \vdots & \vdots & \ddots & 0 \\
a_{1} & a_{2} & a_{3} & \cdots & a_{n}
\end{array}\right) \mid a_{i} \in \mathbb{R}, 1 \leq i \leq n\right\} .
\end{aligned}
$$

Thus $\left\{E_{1}, E_{2}, \cdots, E_{n}\right\}$ can not be a basis for module $M$ over $\mathbb{R}$. However if we take the system of generators $B=\left\{I E_{1}, I E_{2}, \cdots, I E_{n}, \eta E_{1}, \eta E_{2}, \cdots, \eta E_{n}\right.$, $\left.\eta^{2} E_{1}, \eta^{2} E_{2}, \cdots, \eta^{2} E_{n}, \cdots, \eta^{m-1} E_{1}, \eta^{m-1} E_{2}, \cdots, \eta^{m-1} E_{n}\right\}, B$ is obviously a basis of the module $M$ over $\mathbb{R}$. Consequently $M$ is an $m n$-dimensional vector space over $\mathbb{R}$.

From Proposition 8 we have seen that for every $X=\left(x_{i j}\right) \in \mathbb{R}_{n}^{m}, X$ can be written as follows:

$$
\begin{aligned}
X= & \left(\begin{array}{ccccc}
x_{11} & x_{12} & x_{13} & \cdots & x_{1 n} \\
x_{21} & x_{22} & x_{23} & \cdots & x_{2 n} \\
\vdots & \vdots & \vdots & \ddots & \vdots \\
x_{m 1} & x_{m 2} & x_{m 3} & \cdots & x_{m n}
\end{array}\right)=\left(\begin{array}{cccc}
x_{m 1} & x_{(m-1) 1} & \cdots & x_{11} \\
0 & x_{m 1} & \cdots & x_{21} \\
\vdots & 0 & \ddots & \vdots \\
0 & \cdots & 0 & x_{m 1}
\end{array}\right) E_{1} \\
& +\left(\begin{array}{cccc}
x_{m 2} & x_{(m-1) 2} & \cdots & x_{12} \\
0 & x_{m 2} & \cdots & x_{22} \\
\vdots & 0 & \ddots & \vdots \\
0 & \cdots & 0 & x_{m 2}
\end{array}\right) E_{2}+\cdots \\
& +\left(\begin{array}{cccc}
x_{m n} & x_{(m-1) n} & \cdots & x_{1 n} \\
0 & x_{m n} & \cdots & x_{2 n} \\
\vdots & 0 & \ddots & \vdots \\
0 & \cdots & 0 & x_{m n}
\end{array}\right) E_{n} .
\end{aligned}
$$

Here by using

$$
\left(\begin{array}{cccc}
x_{m k} & x_{(m-1) k} & \cdots & x_{1 k} \\
0 & x_{m k} & \cdots & x_{2 k} \\
\vdots & 0 & \ddots & \vdots \\
0 & \cdots & 0 & x_{m k}
\end{array}\right)=x_{m k} \eta_{0}+x_{(m-1) k} \eta_{1}+\cdots+x_{1 k} \eta_{m-1}
$$

$X$ also can be expressed as follows:

$$
\begin{gathered}
X=\left(x_{m 1} \eta_{0}+x_{(m-1) 1} \eta_{1}+\cdots+x_{11} \eta_{m-1}\right) E_{1}+\left(x_{m 2} \eta_{0}+x_{(m-1) 2} \eta_{1}+\cdots+\right. \\
\left.x_{12} \eta_{m-1}\right) E_{2}+\cdots+\left(x_{m n} \eta_{0}+x_{(m-1) n} \eta_{1}+\cdots+x_{1 n} \eta_{m-1}\right) E_{n} .
\end{gathered}
$$


The set $M=\mathbb{R}_{n}^{m}$ is a vector space over the field $\mathbb{R}$. If $m \neq n$ for the matrices $A_{m \times n}$ and $B_{m \times n}$ multiplication is not defined but if $m=n$ then $M$ is an associative algebra with identity. Furthermore if we use a commutative ring with identity then $M=\mathbb{R}_{n}^{m}$ is a module over the ring $R$. Multiplication is defined when $m=n$, so the operation is associative and it has unity.

Theorem 9. Let $\boldsymbol{A}$ be a local ring with a maximal ideal $J$ and $M=\boldsymbol{A}^{n}$. Then for $u_{1}, u_{2}, \cdots, u_{k} \in \boldsymbol{A} \backslash J$ and $x_{i j} \in J$, there are linearly independent vectors such that $\alpha_{1}=\left(u_{1}, x_{21}, x_{31}, \cdots, x_{n 1}\right), \alpha_{2}=\left(x_{12}, u_{2}, x_{32}, \cdots, x_{n 2}\right), \alpha_{3}=\left(x_{13}\right.$, $\left.x_{23}, u_{3}, \cdots, x_{n 3}\right), \cdots, \alpha_{k}=\left(x_{1 k}, x_{2 k}, x_{3 k}, \cdots, u_{k}, x_{(k+1) k}, \cdots, x_{n k}\right)$, and for $k=n$, the set $\left\{\alpha_{1}, \alpha_{2}, \cdots, \alpha_{n}\right\}$ is a basis for $M$.

Proof. First let $k=1$ so we deal with only $\alpha_{1}=\left(u_{1}, x_{21}, x_{31}, \cdots, x_{n 1}\right) \in M$.

For $a_{1} \in \boldsymbol{A}$, let $a_{1} \alpha_{1}=0 \Rightarrow a_{1} \alpha_{1}=\left(a_{1} u_{1}, a_{1} x_{21}, a_{1} x_{31}, \cdots, a_{1} x_{n 1}\right)=$ $(0,0,0, \cdots, 0)$, then from the equality of first components, we get $a_{1} u_{1}=0$. Since $u_{1}$ is a unit, we obtain $a_{1}=0$. Thus the vector $\alpha_{1}$ is linearly independent.

Let $k=2$ so we deal with

$$
\alpha_{1}=\left(u_{1}, x_{21}, x_{31}, \cdots, x_{n 1}\right) \text { and } \alpha_{2}=\left(x_{12}, u_{2}, x_{32}, \cdots, x_{n 2}\right) .
$$

Then for $a_{1}, a_{2} \in \boldsymbol{A}$, let

$$
\begin{aligned}
a_{1} \alpha_{1}+a_{2} \alpha_{2} & =0 \Rightarrow a_{1}\left(u_{1}, x_{21}, x_{31}, \cdots, x_{n 1}\right)+a_{2}\left(x_{12}, u_{2}, x_{32}, \cdots, x_{n 2}\right) \\
& =(0,0,0, \cdots, 0) .
\end{aligned}
$$

From the equality of first two components, we get $a_{1} u_{1}+a_{2} x_{12}=0, a_{1} x_{21}+$ $a_{2} u_{2}=0$. If we compose these equations, we obtain

$$
a_{1}=-a_{2} x_{12} u_{1}^{-1}
$$

and

$$
a_{2}\left(u_{2}-x_{12} u_{1}^{-1} x_{21}\right)=0
$$

Since $u_{2} \in \boldsymbol{A} \backslash J$ and $x_{12} u_{1}^{-1} x_{21} \in J$, we have $u_{2}-x_{12} u_{1}^{-1} x_{21} \in \boldsymbol{A} \backslash J$, so we find $a_{2}=0$ from the equation (2). If we put $a_{2}=0$ in equation (1), we get $a_{1}=0$. Thus the vectors $\alpha_{1}$ and $\alpha_{2}$ are linearly independent.

Finally to understand the process, let $k=3$ so we have deal with $a_{1}=\left(u_{1}\right.$, $\left.x_{21}, x_{31}, \cdots, x_{n 1}\right), \alpha_{2}=\left(x_{12}, u_{2}, x_{32}, \cdots, x_{n 2}\right), \alpha_{3}=\left(x_{13}, x_{23}, u_{3}, \cdots, x_{n 3}\right) \in$ $M$. Let $a_{1} \alpha_{1}+a_{2} \alpha_{2}+a_{3} \alpha_{3}=0$. From the equality of first three components, we obtain the following equations:

$$
\begin{aligned}
& a_{1} u_{1}+a_{2} x_{12}+a_{3} x_{13}=0 \\
& a_{1} x_{21}+a_{2} u_{2}+a_{3} x_{23}=0 \\
& a_{1} x_{31}+a_{2} x_{32}+a_{3} u_{3}=0 .
\end{aligned}
$$


We get

$$
a_{1}=-\left(a_{2} x_{12}+a_{3} x_{13}\right) u_{1}^{-1}
$$

from equation (3). Then if we put $a_{1}$ in equation (4), we obtain $a_{2}\left(-x_{12} u_{1}^{-1} x_{21}+\right.$ $\left.u_{2}\right)+a_{3}\left(-x_{13} u_{1}^{-1} x_{21}+x_{23}\right)=0$.

Since $x_{12} u_{1}^{-1} x_{21} \in J$ and $u_{2} \in \boldsymbol{A} \backslash J$, we get $x_{12} u_{1}^{-1} x_{21}+u_{2} \in \boldsymbol{A} \backslash J$ from which we obtain

$$
a_{2}=a_{3}\left(x_{13} u_{1}^{-1} x_{21}-x_{23}\right)\left(-x_{12} u_{1}^{-1} x_{21}+u_{2}\right)^{-1} .
$$

Now if we put $a_{1}$ and $a_{2}$ in equation (5) and compose the equations we get

$$
a_{3}\left[\begin{array}{c}
\left(x_{23}-x_{13} u_{1}^{-1} x_{21}\right)\left(u_{2}-x_{12} u_{1}^{-1} x_{21}\right) x_{12} u_{1}^{-1} x_{31}-x_{13} u_{1}^{-1} x_{31} \\
+\left(x_{13} u_{1}^{-1} x_{21}-x_{23}\right)\left(u_{2}-x_{12} u_{1}^{-1} x_{21}\right)^{-1} x_{32}+u_{3}
\end{array}\right]=0 .
$$

Since $\left(x_{23}-x_{13} u_{1}^{-1} x_{21}\right)\left(u_{2}-x_{12} u_{1}^{-1} x_{21}\right) x_{12} u_{1}^{-1} x_{31}-x_{13} u_{1}^{-1} x_{31}+\left(x_{13} u_{1}^{-1} x_{21}-\right.$ $\left.x_{23}\right)\left(u_{2}-x_{12} u_{1}^{-1} x_{21}\right)^{-1} x_{32}+u_{3} \in \boldsymbol{A} \backslash J$, we obtain $a_{3}=0$. By using $a_{3}=0$ in equations (6) and (7), we get $a_{2}=0$ and $a_{1}=0$. Thus the vectors $\alpha_{1}, \alpha_{2}$ and $\alpha_{3}$ are linearly independent.

For a positive integer $k$, we have

$$
\begin{aligned}
& \alpha_{1}=\left(u_{1}, x_{21}, x_{31}, \cdots, x_{n 1}\right), \alpha_{2}=\left(x_{12}, u_{2}, x_{32}, \cdots, x_{n 2}\right), \\
& \alpha_{3}=\left(x_{13}, x_{23}, u_{3}, \cdots, x_{n 3}\right), \cdots, \alpha_{k}=\left(x_{1 k}, x_{2 k}, \cdots, u_{k}, \cdots, x_{n k}\right) .
\end{aligned}
$$

Let $\sum_{i=1}^{k} a_{i} \alpha_{i}=0$. Then from the equality of first $k$ components to zero, we get exactly $k$ equations. By similar calculations, we obtain all $a_{i}$ 's to be zero. Thus the set $\left\{\alpha_{1}, \alpha_{2}, \ldots, \alpha_{k}\right\}$ is linearly independent.

Finally we express Remark 2 from [3] as a proposition and we give detailed proof of this proposition.

Proposition 10. An $\boldsymbol{A}$-module $M$ over a local ring $\boldsymbol{A}$ is an $\boldsymbol{A}$-space if and only if it is a free finitely dimensional module.

Proof. Let $M$ be a free module over $\boldsymbol{A}$ with a basis $\left\{E_{1}, E_{2}, \cdots, E_{n}\right\}$. Now we will show that the $\boldsymbol{A}$-module $M$ is an $\boldsymbol{A}$-space. For every $\beta \in M, \beta$ can be written as

$$
\beta=b_{1} E_{1}+b_{2} E_{2}+\cdots+b_{n} E_{n} \in \boldsymbol{A} E_{1}+\boldsymbol{A} E_{2}+\cdots+\boldsymbol{A} E_{n} .
$$

Let $\beta \in \sum_{i \neq j} \boldsymbol{A} E_{i} \cap \boldsymbol{A} E_{j}$. Then we can write

$$
\beta=a_{1} E_{1}+a_{2} E_{2}+\cdots+a_{j-1} E_{j-1}+a_{j+1} E_{j+1}+\cdots+a_{n} E_{n}=a_{j} E_{j} .
$$


So we get $a_{1} E_{1}+a_{2} E_{2}+\cdots+a_{j-1} E_{j-1}-a_{j} E_{j}+a_{j+1} E_{j+1}+\cdots+a_{n} E_{n}=0$. Here for $1 \leq i \leq n$, all $a_{i}$ 's must be zero because of the linearly independence of the vectors $E_{1}, E_{2}, \cdots, E_{n}$. Thus $\beta$ must be zero. Then since the following conditions are satisfied:

$$
\begin{aligned}
& \text { i) } M=\boldsymbol{A} E_{1}+\boldsymbol{A} E_{2}+\cdots+\boldsymbol{A} E_{n} \\
& \text { ii) } \sum_{i \neq j} \boldsymbol{A} E_{i} \cap \boldsymbol{A} E_{j}=\{0\} .
\end{aligned}
$$

We can say $M=\boldsymbol{A} E_{1} \oplus \boldsymbol{A} E_{2} \oplus \cdots \oplus \boldsymbol{A} E_{n}$. Now we show that for $1 \leq i \leq n$, all functions $f_{i}: \boldsymbol{A} \rightarrow \boldsymbol{A} E_{i}$ are isomorphisms. For every $X, Y \in \boldsymbol{A}$,

$$
f_{i}(X+Y)=(X+Y) E_{i}=X E_{i}+Y E_{i}=f_{i}(X)+f_{i}(Y)
$$

and also for every $X \in \boldsymbol{A}$ and for every $\lambda \in \mathbb{R}$,

$$
f_{i}(\lambda X)=(\lambda X) E_{i}=\lambda\left(X E_{i}\right)=\lambda f_{i}(X)
$$

So each $f_{i}$ preserves the operations. Let $f_{i}(X)=f_{i}(Y)$ for some $X, Y \in \boldsymbol{A}$. So we get $X E_{i}=Y E_{i}$ and hence $(X-Y) E_{i}=0$. By using the linear independence of the vectors $E_{i}$ for $1 \leq i \leq n$,

$$
X-Y=0 \text { and hence } X=Y
$$

is obtained. Thus for $1 \leq i \leq n$, every $f_{i}$ is one-to-one. For every $X E_{i} \in \boldsymbol{A} E_{i}$, there is obviously an $X \in \boldsymbol{A}$ such that $f(X)=X E_{i}$. Thus for $1 \leq i \leq n$, every $f_{i}$ is onto.

Conversely let $M$ be a finite dimensional $\boldsymbol{A}$-space over the local ring $\boldsymbol{A}$. Now we show that $M$ is a finite dimensional free module. Since $M$ is an $\boldsymbol{A}$-space, there are the vectors $E_{1}, E_{2}, \cdots, E_{n}$, and they form a finite basis of $M$. So $M$ is a finite dimensional free module.

Acknowledgment. This work, a part of $\mathrm{PhD}$ thesis of first author, is supported by The Research Fund of University of Uludag Project number $\operatorname{KUAP}(\mathrm{F})$ $2012 / 56$.

\section{References}

[1] B. R. McDonald, Geometric algebra over local rings, Marcel Dekker, New York, 1976.

[2] M. Jukl, Linear forms on free modules over certain local ring, Acta Univ. Palack. Olomuc. Fac. Rerum Natur. Math., 32 (1993), 49-62. 
[3] M. Jukl, Grassmann formula for certain type of modules, Acta Univ. Palack. Olomuc. Fac. Rerum Natur. Math., 34 (1995), 69-74.

[4] T. W. Hungerford, Algebra, Holt, Rinehart and Winston, New York, 1974.

[5] K. Nomizu, Fundamentals of Linear Algebra, McGraw-Hill, New York, 1966.

Fatma Özen ERDOĞAN, Süleyman ÇİTÇi, Atilla AKPINAR

Department of Mathematics

Faculty of Sceince,

Uludag University, Bursa, Turkey

Emails: fatmaozen@uludag.edu.tr, sciftci@uludag.edu.tr, aakpinar@uludag.edu.tr 